\title{
Subitizing and similarity: Toward a pattern-matching theory of enumeration
}

\author{
GORDON D. LOGAN and N. JANE ZBRODOFF \\ Vanderbilt University, Nashville, Tennessee
}

\begin{abstract}
Pattern-matching theories of subitizing claim that subjects enumerate displays with small numerosities by retrieving numerical responses associated with similar displays experienced in the past. Such retrieval implies that displays with small numerosities are similar to other displays of the same numerosity and dissimilar to other displays of different numerosities. These hypotheses were tested by having subjects rate the similarities of displays of dot patterns with numerosities in the range of $1-10$. One group of subjects rated patterns of the same numerosity. Their ratings were higher for patterns in the subitizing range (numerosities of 1-3) than for patterns beyond the subitizing range (numerosities of 4-10). Another group rated patterns of different numerosities. Their ratings were lower in the subitizing range than beyond the subitizing range. An analysis based on multidimensional scaling suggested that numerosity could be retrieved accurately for displays of 1-3 dots, but not for displays of $4-10$ dots.
\end{abstract}

When mathematically literate young adults enumerate displays of clearly perceptible objects, they appear to engage in two phenomenologically different processes. If the displays contain four or more objects that are exposed until the subject responds, subjects enumerate by counting, which involves iterative stepping through the display and establishing a one-to-one correspondence between objects and numbers until all of the objects have been indexed. If the displays contain one to three or four objects, subjects appear to apprehend the numerosity immediately through a process called subitizing - they "see" the numerosity directly without having to count (Kaufman, Lord, Reese, \& Volkmann, 1949; see Cattell, 1886; Jevons, 1871; Warren, 1897; Wundt, 1896). Subitizing has been observed in other tasks, such as numerosity discrimination (Folk, Egeth, \& Kwak, 1988), and it has been observed in preverbal infants (Spelke, 2000; Starkey \& Cooper, 1980) and animals (Davis \& Pérusse, 1988), but the focus of this article is on subitizing in the enumeration task with mathematically literate adults. In this context, subitizing is much faster than counting. With displays of one to three or four objects, reaction time is largely unaffected by numerosity, increasing by 10 or $20 \mathrm{msec}$ per item. With larger displays, it increases by 300 or $400 \mathrm{msec}$ per item (Jensen, Reese, \& Reese, 1950; Klahr, 1973; Mandler \& Shebo, 1982; Saltzman \& Garner,

This research was supported by Grants SBR 9808971 and 0133202 from the National Science Foundation. We are grateful to Julie Delheimer for testing the subjects and to Tom Palmeri for help with the multidimensional scaling. Correspondence concerning this article may be addressed to G. D. Logan or N. J. Zbrodoff, Department of Psychology, Vanderbilt University, Nashville, TN 37203 (e-mail: gordon.logan@ vanderbilt.edu or jane.zbrodoff@ vanderbilt.edu).
1948; Trick \& Pylyshyn, 1994). Subitizing and counting have been dissociated in several ways (Dehaene \& Cohen, 1994; Sathian et al., 1999; Simon \& Vaishnavi, 1996; Sliwinski, 1997; Trick, Enns, \& Brodeur, 1996; Tuholski, Engle, \& Baylis, 2001; but see Balakrishnan \& Ashby, 1991, 1992). Several theories have been advanced to explain subitizing (Dehaene \& Changeux, 1993; Gallistel \& Gelman, 1991; Klahr, 1973; Trick \& Pylyshyn, 1994; van Oeffelen \& Vos, 1982). This article is concerned with theories that explain subitizing in terms of pattern matching (Mandler \& Shebo, 1982; Peterson \& Simon, 2000; Wolters, van Kempen, \& Wijlhuizen, 1987).

Pattern-matching theories assume that subjects retrieve the numerosity of a display from memory without engaging a counting process. The display acts as a retrieval cue that activates numerical responses that were previously associated with similar displays. If the activation is sufficiently high, the numerical response that is retrieved can be given as a response in an enumeration task (see Logan, 1988). Activation depends on the similarity of the current display to previous displays with the same and different numerosities. Activation is higher the higher the similarity to displays of the same numerosity and the lower the similarity to displays of different numerosities (Nosofsky, 1984, 1988). The pattern-matching hypothesis assumes that displays in the subitizing range (one to three or four objects) can support accurate retrieval of numerosity, whereas displays in the counting range (more than four objects) cannot and so must be enumerated by iterative counting. Thus, displays of a given numerosity in the subitizing range must be more similar to other displays of the same numerosity and less similar to displays of different numerosities than are displays of a given numerosity in the counting range. The experiment reported here was designed to test these hypotheses. 
The literature provides some support for the patternmatching hypothesis. Mandler and Shebo (1982) argued that displays in the subitizing range resemble canonical, or prototypical, patterns. For example, displays of one object look alike except for the location of the objects. Displays of two objects form a virtual line that can differ in location, orientation, or both. Displays of three objects can form a line, which can differ in location, orientation, or both, or a triangle, which can differ in aspect ratio, location, orientation, or some combination of these features. The larger the number of objects in the display, the more ways there are for the displays to differ from each other. Consistent with their analysis, Mandler and Shebo showed that familiar patterns, such as the dot patterns on dice, can be enumerated very quickly, even when the numerosity is beyond the subitizing range (also see Dehaene \& Cohen, 1994; Wender \& Rothkegel, 2000).

Canonical patterns, or prototypes, are not necessary to support the kind of memory process that patternmatching theories propose. Collections of instances may suffice instead (e.g., Logan, 1988), as long as instances of small numerosities are similar to other instances of the same numerosity and dissimilar to instances of different numerosities. Indeed, subjects can learn to enumerate patterns with large numerosities (6-11 objects) at a rate comparable to the 10 or $20 \mathrm{msec}$ per item rate observed in the subitizing range if they are given enough practice with specific patterns (Lassaline \& Logan, 1993; Palmeri, 1997; Wolters et al., 1987). Palmeri showed that subjects could transfer this fast enumeration to novel patterns that were highly similar to trained patterns. Thus, subitizing may depend on retrieval of similar instances of small numerosities.

Peterson and Simon (2000) argued that patterns with small numerosities were more likely to recur in enumeration tasks than were patterns with large numerosities, so small patterns should support retrieval more readily than do large patterns. They supported their argument by counting the number of unique patterns in grids of $M$ cells. The number of unique patterns of size $N$ (i.e., in which no more than one object appears in one cell) is $M ! /[N !(M-N) !]$, which grows rapidly as $N$ increases. Thus, there are fewer patterns with small numerosities. Given equal exposure to patterns of different numerosities, patterns with small numerosities would repeat more often and would be more likely to be learned well enough to support retrieval. Peterson and Simon trained a version of Anderson's (1993) ACT-R model and several parallel distributed processing models on patterns with numerosities ranging from one to eight and found that patterns of one to three were learned much faster than patterns with larger numerosities.

The pattern-matching hypothesis assumes that (1) patterns with small numerosities are more similar to other patterns of the same numerosity than are patterns with large numerosities and (2) patterns with small numerosities are less similar to other patterns with different nu- merosity than are patterns with large numerosities. Small numerosity patterns have greater within-category similarity and less between-category similarity, whereas large numerosity patterns have less within-category similarity and more between-category similarity. The purpose of the present article was to test these hypotheses. We report an experiment in which one group of subjects enumerated patterns of 1-10 objects and two groups of subjects rated the similarity of the appearance of patterns generated by the same program. The same-numerosity group rated the similarity of two patterns with the same numerosity, and the different-numerosity group rated the similarity of two patterns with different numerosity. If the pattern-matchinghypothesis is right, the same-numerosity group should give higher similarity ratings to patterns in the subitizing range, and the different-numerosity group should give lower similarity ratings to patterns in the subitizing range.

Alternative theories of subitizing are mute on the relation between similarity of appearance and subitizing. Theories based on magnitude representation say nothing about similarity of appearance (e.g., Gallistel \& Gelman, 1991; Meck \& Church, 1983; van Oeffelen \& Vos, 1982). Indeed, Dehaene and Changeux's (1993) magnitudebased theory assumes that patterns are normalized before their magnitudes are estimated, so it would predict no effect of similarity. Trick and Pylyshyn's (1994) theory assumes that subitizing is done by spatial indices that are preattentive and limited in number. Counting is required when a display contains more objects than the number of spatial indices. Trick and Pylyshyn said nothing about the role of similarity in subitizing, except that the objects should be available preattentively. Klahr's (1973) production system for subitizing assumes a rapid serial application of productions. Klahr said nothing about the role of similarity in subitizing, except that subitizable patterns should contain four or fewer objects.

\section{METHOD}

\section{Subjects}

The experiment employed three groups of 12 subjects each. One group enumerated the dot patterns, and two groups rated the similarity of dot patterns. All 36 subjects were students from psychology courses, who participated to fulfill course requirements.

\section{Apparatus and Stimuli}

The stimuli were displayed on Gateway 2000 Crystalscan 1024 NI monitors controlled by Gateway 2000486 computers. The stimuli were sets of 1-10 white asterisks ("dots") on a black background that appeared in random positions in an $8 \times 8$ grid. The grid was $10.69 \mathrm{~cm}$ high and $7.25 \mathrm{~cm}$ wide. Viewed at a distance of $60 \mathrm{~cm}$, the grid subtended $10.10^{\circ}$ of visual angle vertically and $6.89^{\circ}$ of visual angle horizontally. Each asterisk was $0.29 \mathrm{~cm}$ high and $0.29 \mathrm{~cm}$ wide, subtending $0.28^{\circ} \times 0.28^{\circ}$ of visual angle. In the enumeration task, one set of asterisks was displayed on each trial, centered on the display screen. In the same-numerosity similarity-rating task, two sets of asterisks with the same numerosity were presented on each trial, one on each side of a white line drawn down the center of the display screen. In the different-numerosity similarity-rating task, 
two sets of asterisks with different numerosities were presented on each trial, one on each side of the white line in the center of the display. A fixation display containing a centered plus sign (+) was presented at the beginning of each trial. After a 500-msec exposure, it was extinguished and replaced immediately by the dot pattern for the enumeration or similarity-rating task, which remained on the screen until the subject responded. After the subject's response, the display went blank for a $1,000-\mathrm{msec}$ intertrial interval. The subjects in the enumeration task responded by speaking into a microphone attached to a voice-activated relay, which stopped the computer's clock. An experimenter, present in the room with the subject, typed the subject's response into the keyboard. The subjects in the similarityrating tasks responded by pressing one of nine numeric keys (1-9) on the top row of the computer's keyboard.

\section{Procedure}

Enumeration task. The enumeration task involved 40 trials with each of 10 numerosities, for a total of 400 trials. The displays were presented in random order, with the constraint that 10 displays of each numerosity occurred in each set of 100 trials. The subjects were told to say the number representing each display's numerosity into the microphone attached to the voice-activated relay as quickly as possible without making errors and loudly enough to cause the display to disappear. The experimenter was present in the room to type the subject's response into the computer and to ensure compliance with the instructions. The subjects were allowed brief breaks every 50 trials.

Similarity-rating task: same numerosity. The same-numerosity similarity-rating task involved 40 trials with each of 10 numerosities, for a total of 400 trials. The displays were presented in random order, subject to the same constraints as the enumeration task. The two patterns in each display had the same numerosity. The subjects were told to rate the similarity of the appearance of the patterns on the left and right sides of the screen on a scale from 1 to 9 , with 1 representing very dissimilar and 9 representing very similar. They were encouraged to use the whole range of the scale. They were told that time was not important (i.e., there was no speed stress, as there was in the enumeration task). The experimenter was not present in the room while they were tested. As in the enumeration task, the subjects were allowed brief breaks every 50 trials.

Similarity-rating task: different numerosity. The differentnumerosity similarity-rating task was the same as the samenumerosity task, except that the two patterns displayed on each trial had different numerosities. There were 12 trials with each of the 45 possible combinations of different numerosities, ranging from 1 to 10 , for a total of 540 trials. For half of the trials, the pattern with the larger numerosity was on the left. For the other half, the pattern with the larger numerosity was on the right. The subjects rated the similarity of the appearance of the patterns on the left and the right sides on the same 1-9 scale. The experimenter was not in the room during testing, and the subjects were allowed brief rests every 50 trials.

\section{RESULTS}

\section{Enumeration Task}

Mean reaction times across subjects for correct responses are plotted as a function of numerosity in panel A of Figure 1. Reaction time was relatively unaffected by numerosity in the range of 1-3 but was strongly affected in the range of 4-10. A one-way analysis of variance (ANOVA) performed on the reaction times showed a highly significant effect of numerosity $[F(9,99)=$ $\left.251.26, M S_{\mathrm{e}}=29,528.25, p<.01\right]$.

We fit linear functions relating reaction time to numerosity within and beyond the subitizing range. For
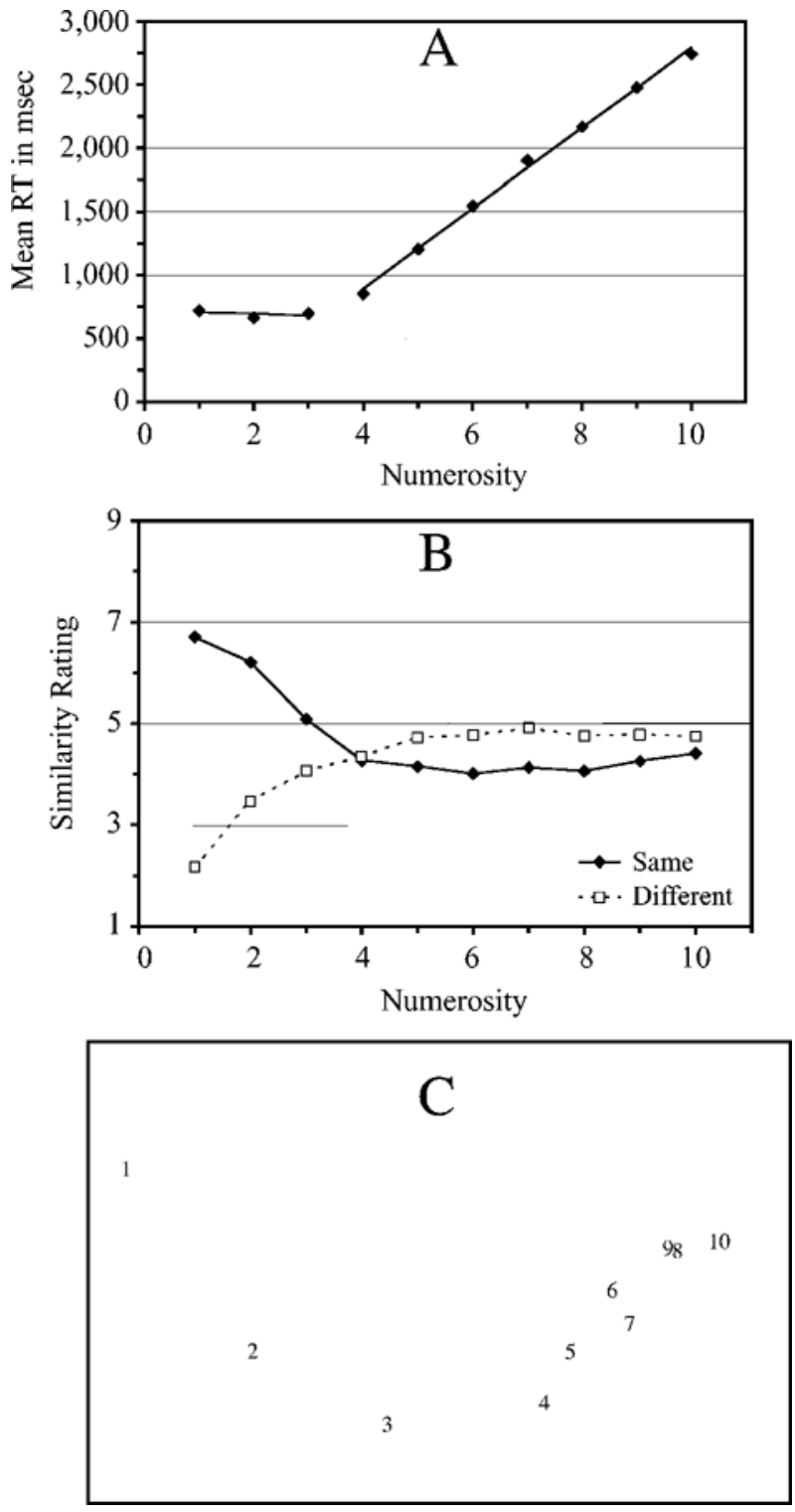

Figure 1. (A) Mean reaction time as a function of numerosity. The points represent the observed data. The lines represent linear regression functions relating reaction time to numerosity within the subitizing range (1-3) and the counting range (4-10). (B) Mean similarity ratings as a function of numerosity for subjects who judged pairs of patterns with the same numerosity and subjects who judged pairs of patterns with different numerosity. (C) Two-dimensional solution for multidimensional similarity scaling of the similarity ratings from subjects who judged pairs of patterns with different numerosity. The numbers represent the location in similarity space of patterns with the corresponding numerosity.

numerosities of $1-3$, the regression equation was RT = $718-11.5 N, r=-.401$, where $N$ is numerosity. For numerosities of $4-10$, the regression equation was $\mathrm{RT}=$ $315.6 N-366, r=.999$. These findings replicate standard results in the literature (e.g., Mandler \& Shebo, 
1982). They suggest that displays of 1-3 objects were subitized, whereas displays of 4-10 objects were counted.

Accuracy varied with numerosity. For numerosities of $1-10$, the probability of a correct response was .975 , $.965, .963, .952, .940, .908, .898, .806, .825$, and .798 , respectively. A one-way ANOVA on the probabilities of correct responses yielded a significant effect of numerosity $\left[F(9,99)=15.34, M S_{\mathrm{e}}=0.003, p<.01\right]$. A contrast comparing the subitizing range of $1-3$ with the counting range of $4-10$ was highly significant $[F(1,99)=$ $56.93, p<.01]$. The decrease in accuracy with increasing numerosity was predictable from a serial-counting model that assumed a constant probability of error for each step in the counting sequence. The counting model predicts that accuracy for numerosity $N$ should equal the probability of correctly executing each counting step raised to the $N$ th power. The probability of correctly executing each step can be estimated by taking the $N$ th root of the observed probability of responding correctly to a display of $N$ elements. For numerosities of $1-10$, the $N$ th root of the probability correct was $.975, .982, .988, .988$, $.988, .984, .982, .973, .979$, and .978 , respectively. These estimates of the probability of responding correctly on each step are relatively constant across numerosities, as the serial-counting model predicts.

\section{Similarity-Rating Tasks}

Same numerosity. The mean similarity ratings for same-numerosity displays were averaged across subjects and plotted as a function of numerosity in panel B of Figure 1. Similarity was rated higher for numerosities within the subitizing range than for numerosities in the counting range. Similarity decreased with numerosity within the subitizing range (1-3) but was relatively unaffected by numerosity in the counting range (4-10). We performed a one-way ANOVA on the similarity ratings to test the significance of these differences. The effect of numerosity was significant $\left[F(9,99)=21.37, M S_{\mathrm{e}}=\right.$ $0.526, p<.01]$. We compared similarity ratings within the subitizing range (1-3) with those in the counting range (4-10) in two ways. First, we computed a contrast comparing similarity ratings in the subitizing range (mean rating $=6.00$ ) with those in the counting range (mean rating $=4.18)$. The contrast was highly significant $[F(1,99)=$ $158.52, p<.01]$. Second, we computed Fisher's least significant difference (LSD) test for $p<.05$ and found a critical difference of .59. By this criterion, the ratings for all the numerosities in the subitizing range were significantly higher than the ratings for all the numerosities in the counting range. The rating for a numerosity of 1 $(M=6.71)$ was significantly higher than the rating for a numerosity of $3(M=5.09)$, and the rating for a numerosity of $2(M=6.21)$ was significantly higher than the rating for a numerosity of 3 . None of the ratings for numerosities beyond the subitizing range (4-10) was different from the others (range $=4.01-4.41$ ) .

Different numerosity. The mean similarity ratings for different-numerosity displays were analyzed in two ways. First, in order to compare the different-numerosity ratings with the same-numerosity ratings, we calculated the average similarity rating for each numerosity. These means reflect how similar a pattern of a given numerosity is to patterns with different numerosities. The means across subjects are plotted as a function of numerosity in panel B of Figure 1. The different-numerosity similarities were the mirror image of the same-numerosity similarities. Different-numerosity similarity was lower for patterns in the subitizing range (1-3) than for patterns in the counting range (4-10). Different-numerosity similarity increased with numerosity in the subitizing range but was relatively unaffected by numerosity in the counting range. These conclusions were confirmed in a oneway ANOVA on the similarity ratings. The effect of numerosity was highly significant $\left[F(9,99)=83.73, M S_{\mathrm{e}}=\right.$ $0.107, p<.01]$. A contrast comparing ratings in the subitizing range $(1-3 ; M=3.23)$ with those in the counting range $(4-10 ; M=4.72)$ was highly significant $[F(1,99)=523.43, p<.01]$. Fisher's LSD for $p<.05$ was .27 . By this criterion, all the ratings in the subitizing range (1-3) were smaller than all the ratings in the counting range (4-10). Within the subitizing range, each of the ratings differed significantly from each other $(M \mathrm{~s}=2.17$, 3.47 , and 4.06 for numerosities of $1-3$, respectively). Within the counting range, the rating for a numerosity of $4(M=4.35)$ differed significantly from the ratings for numerosities of 5-10, which did not differ significantly from each other (range $=4.73-4.91)$.

Our second analysis took a first step toward modeling the enumeration task, asking whether the similarities in the subitizing range could support retrieval while the similarities in the counting range could not. To answer this question, we generated retrieval probabilities from Nosofsky's (1984, 1988) generalized context model (GCM), which is formally related to the instance theory (Logan, 2002). If retrieval can support enumeration in the subitizing range, but not in the counting range, the estimated retrieval probabilities should be high in the subitizing range and low in the counting range. To test this prediction, we performed a multidimensional scaling analysis on the ratings from the different-numerosity group, averaged across subjects within numerosities. One- and two-dimensional solutions had sufficiently low stress values (.044 and .025 , respectively), and the estimated distances correlated highly with the similarity ratings (.995 and .998, respectively). The two-dimensional solution is plotted in panel C of Figure 1. We used these distances to estimate theoretical similarities in GCM, using the GCM's assumption that similarity is an exponential function of distance:

$$
\eta(x, i)=\exp \left(-s \cdot d_{x i}\right),
$$

where $s$ is a sensitivity parameter. Distance was defined by a Euclidean metric

$$
d_{x i}=\left[\left(x_{1}-i_{1}\right)^{2}+\left(x_{2}-i_{2}\right)^{2}\right]^{\frac{1}{2}}
$$

(Shin \& Nosofsky, 1992). 
In our application of GCM, we assumed that the subjects chose a response to a given display by comparing it with representations of displays of all possible numerosities according to GCM's choice rule. The choice of a response depends on the ratio of the similarity of the display to a representation of a given numerosity (e.g., $i$ ) to the sum of the similarity of the display to representations of all 10 numerosities. That is,

$$
P(x \mid i)=\frac{\eta(x, i) \beta_{i}}{\sum_{j=1}^{10} \eta(x, j) \beta_{j}},
$$

where $\beta_{i}$ is the bias for category $i$. Because all numerosities were equally frequent and all responses were equally valid, we assumed that all biases were equal to 1 . We calculated the probability of responding with each numerosity from 1 to 10 to displays of numerosities of $1-10$, with $s=3$. The probabilities appear in Table 1 . The table shows that the mean probability of retrieving the correct numerosity was .955 in the subitizing range (1-3) and .491 in the counting range (4-10). Accuracies above .9 are sufficient to support retrieval as a processing strategy. Similar results were obtained by applying GCM to the one-dimensional solution.

\section{DISCUSSION}

The enumeration task replicated standard results. Enumeration was fast and relatively unaffected by numerosity in the subitizing range (1-3), and it was slow and strongly affected by numerosity beyond the subitizing range (4-10). In agreement with the similarity hypotheses derived from pattern-matching theories, patterns of a given numerosity in the subitizing range were rated as more similar to other patterns of the same numerosity and as less similar to patterns of different numerosities than were patterns of a given numerosity beyond the subitizing range. Put differently, within-category similarity was high and between-category similarity was low in the subitizing range, whereas within-category simi- larity was low and between-category similarity was high in the counting range. Thus, displays in the subitizing range are likely to retrieve a correct numerosity response, whereas displays in the counting range are not likely to retrieve a correct numerosity response and must be enumerated by counting. This analysis was supported by the application of GCM to the ratings from the differentnumerosity group, which showed retrieval probabilities above .9 in the subitizing range. The GCM analysis gives the probability of single-step, direct-access memory retrieval, which should be fast and automatic according to the instance theory of automaticity (Logan, 1988; Nosofsky \& Palmeri, 1997; Palmeri, 1997).

The GCM analysis is promising, but it is incomplete in two respects. First, it assumes that displays of the same numerosity are equally similar to each other (i.e., it assumes $d_{i i}=0$ for all $i$ ), but the ratings from the samenumerosity group suggest that patterns within the subitizing range are more similar to other patterns of the same numerosity than to patterns in the counting range. A more complete analysis would have to take this difference into account. The difference seems likely to enhance the effects already observed. Second, the GCM analysis represents all patterns of a given numerosity as a single point in multidimensional similarity space, but a more realistic analysis would represent each pattern as a distinct point (e.g., Shin \& Nosofsky, 1992). The similarity ratings suggest that points representing patterns with small numerosities would be clustered together closely in relatively separate regions of similarity space, whereas points representing patterns with large numerosities would be spread farther apart and would be more likely to overlap with adjacent numerosities.

The data support the relations between numerosity and similarity predicted by pattern-matching theories of subitizing, but they do not distinguish between alternative versions of pattern-matching theory. We prefer to interpret them in terms of instance theory and GCM, but it is also possible to interpret them in terms of Mandler and Shebo's (1982) canonical patterns theory and in terms of Anderson's (1993) ACT-R model or the various parallel

Table 1

Retrieval Probabilities as a Function of Numerosity From the Generalized Context Model Analysis of the Similarity Ratings From Subjects Who Rated Pairs of Patterns That Differed in Numerosity

\begin{tabular}{ccccccccccc}
\hline & \multicolumn{10}{c}{ Stimulus } \\
\cline { 2 - 11 } Response & 1 & 2 & 3 & 4 & 5 & 6 & 7 & 8 & 9 & 10 \\
\hline 1 & $\mathbf{. 9 9 3}$ & .007 & .000 & .000 & .000 & .000 & .000 & .000 & .000 & .000 \\
2 & .007 & $\mathbf{. 9 5 5}$ & .035 & .002 & .001 & .000 & .000 & .000 & .000 & .000 \\
3 & .003 & .033 & $\mathbf{. 9 1 6}$ & .030 & .013 & .003 & .003 & .001 & .001 & .000 \\
4 & .000 & .001 & .022 & $\mathbf{. 6 7 4}$ & .194 & .037 & .051 & .008 & .009 & .003 \\
5 & .000 & .001 & .008 & .158 & $\mathbf{. 5 5 0}$ & .106 & .127 & .021 & .020 & .010 \\
6 & .000 & .000 & .002 & .026 & .089 &. $\mathbf{4 6 0}$ & .201 & .087 & .099 & .038 \\
7 & .000 & .000 & .002 & .036 & .108 & .205 &. $\mathbf{4 7 0}$ & .070 & .073 & .036 \\
8 & .000 & .000 & .000 & .004 & .015 & .072 & .057 & $\mathbf{. 3 8 1}$ & .312 & .158 \\
9 & .000 & .000 & .000 & .005 & .014 & .083 & .060 & .317 & $\mathbf{. 3 8 7}$ & .134 \\
10 & .000 & .000 & .000 & .003 & .009 & .042 & .039 & .214 & .178 & $\mathbf{. 5 1 4}$ \\
\hline
\end{tabular}

Note-The probability of retrieving the correct numerosity is in boldface. 
distributed processing models investigated by Peterson and Simon (2000). Future research will be required to distinguish between these alternatives.

Similarity of appearance plays no role in competing theories of subitizing (e.g., Dehaene \& Changeux, 1993; Gallistel \& Gelman, 1991; Klahr, 1973; Meck \& Church, 1983; Trick \& Pylyshyn, 1994; van Oeffelen \& Vos, 1982). The present data and other results supporting the pattern-matching hypothesis (Dehaene \& Cohen, 1994; Lassaline \& Logan, 1993; Mandler \& Shebo, 1982; Palmeri, 1997; Peterson \& Simon, 2000; Wender \& Rothkegel, 2000; Wolters et al., 1987) suggest that a complete account of subitizing in particular and enumeration in general must specify the role of similarity. It may be possible to modify the competing theories to account for similarity. The present data suggest that this should be an important goal for future research.

The role of similarity in the pattern-matching account is similar to the role of magnitude in competing theories of subitizing. Both similarity and magnitude can be applied to the whole range of numerosities, within and beyond the subitizing range. However, constraints on similarity and magnitude representations allow them to support accurate enumeration only in the subitizing range. In our similarity account, only small numerosities are sufficiently distinct from each other to support accurate enumeration. The same is true in magnitude accounts. Either the scale is compressed at large magnitudes (Dehaene \& Changeux, 1993; van Oeffelen \& Vos, 1982), or the variability in the representation increases with magnitude (Gallistel \& Gelman, 1991; Meck \& Church, 1983), so that magnitudes associated with large numerosities are easily confused. Requiring subjects to enumerate accurately forces them to count iteratively beyond the subitizing range, because neither similarity nor magnitude can support accurate enumeration of large numerosities. In this respect, our similarity-based patternmatching account and magnitude accounts are different from approaches such as Klahr's (1973) theory and Trick and Pylyshyn's (1994) theory, which assume that the processes underlying subitizing are restricted to numerosities of one to three or four.

Although similarity and magnitude accounts share the idea of compression of the representation at large values of numerosity, it is important to remember that they address different representations. Our account addresses representations of the similarity of appearance of patterns, whereas the magnitude accounts address representation of magnitude. The similarity representation may be more malleable than the magnitude representation. Several studies have shown rapid enumeration of large patterns (6-11 dots) that were presented repeatedly (Lassaline \& Logan, 1993; Palmeri, 1997; Wolters et al., 1987). Apparently, repetition of the same pattern makes it more distinctive and, therefore, more able to support retrieval of the correct numerosity. These effects are specific to the particular patterns that were repeated and do not generalize to other patterns of the same numerosity
(Lassaline \& Logan, 1993; Palmeri, 1997), so they likely affect the similarity representation, rather than the magnitude representation.

Another important difference between similarity and magnitude accounts is that our similarity account addresses the task of enumeration-giving a number that reflects numerosity-in mathematically literate adults, whereas magnitude accounts address a variety of tasks in a variety of subjects, including preverbal infants and animals with no mathematical literacy. Advocates of the magnitude accounts interpret them as precursors to arithmetic and mathematical knowledge that develop early (Dehaene \& Changeux, 1993; Gallistel \& Gelman, 1991). The similarity account requires knowledge of numbers and the principles of counting and so reflects abilities that appear much later than the abilities described in the magnitude accounts. On the one hand, the magnitude account is more general, and the processes it describes are likely to be in place before subjects begin to learn to match patterns. One could ask whether pattern matching is necessary, given the ability to represent and process magnitudes. On the other hand, the ability to attach arbitrary labels to patterns is ubiquitous and common. It is as likely to be applied to enumeration as to any other task, even if it is redundant with more primitive mechanisms. Indeed, accurate enumeration is part of mathematical literacy, and the enumeration task is likely to encourage association of arbitrary labels (numbers) to patterns.

\section{REFERENCES}

Anderson, J. R. (1993). Rules of the mind. Hillsdale, NJ: Erlbaum. Balakrishnan, J. D., \& Ashby, F. G. (1991). Is subitizing a unique numerical ability? Perception \& Psychophysics, 50, 555-564.

Balakrishnan, J. D., \& Ashby, F. G. (1992). Subitizing: Magical numbers or mere superstition? Psychological Research, 54, 80-90.

CAttell, J. M. (1886). Über die Trägheit der Netzhaut und des Sehcentrums. Philosophische Studien, 3, 94-127.

DAvis, H., \& PÉrusse, R. (1988). Numerical competence in animals: Def initional issues, current evidence, and a new research agenda. $B e$ havioral \& Brain Sciences, 11, 561-615.

Dehaene, S., \& Changeux, J.-P. (1993). Development of elementary numerical abilities: A neuronal model. Journal of Cognitive Neuroscience, 5, 390-407.

Dehaene, S., \& Cohen, L. (1994). Dissociable mechanisms of subitizing and counting: Neuropsychological evidence from simultanagnosic patients. Journal of Experimental Psychology: Human Perception \& Performance, 20, 958-975.

Folk, C. L., Egeth, H., \& Kwak, H.-W. (1988). Subitizing: Direct apprehension or serial processing? Perception \& Psychophysics, 44, 313-320.

Gallistel, C. R., \& Gelman, R. (1991). Subitizing: The preverbal counting process. In W. Kessen, A. Ortony, \& F. I. M. Craik (Eds.), Memories, thoughts and emotions: Essays in honor of George Mandler (pp. 65-81). Hillsdale, NJ: Erlbaum.

Jensen, E. M., Reese, E. P., \& Reese, T. W. (1950). The subitizing and counting of visually presented fields of dots. Journal of Psychology, 30, 363-392.

Jevons, W. S. (1871). The power of numerical discrimination. Nature, 3, 281-282.

Kaufman, E. L., Lord, M. W., Reese, T. W., \& Volkmann, J. (1949). The discrimination of visual number. American Journal of Psychology, 62, 498-525. 
KLAHr, D. (1973). A production system for counting, subitizing, and adding. In W. G. Chase (Ed.), Visual information processing (pp. 527 546). New York: Academic Press.

Lassaline, M. L., \& Logan, G. D. (1993). Memory-based automaticity in the discrimination of visual numerosity. Journal of Experimental Psychology: Learning, Memory, \& Cognition, 19, 561-581.

LogAN, G. D. (1988). Toward an instance theory of automatization. Psychological Review, 95, 485-491.

Logan, G. D. (2002). An instance theory of attention and memory. Psychological Review, 109, 376-400.

Mandler, G., \& Shebo, B. J. (1982). Subitizing: An analysis of its component processes. Journal of Experimental Psychology: General, 111, 1-22.

Meck, W. H., \& Church, R. M. (1983). A mode control model of counting and timing processes. Journal of Experimental Psychology: Animal Behavior Processes, 9, 320-334.

NosofsKy, R. M. (1984). Choice, similarity, and the context theory of classif ication. Journal of Experimental Psychology: Learning, Memory, \& Cognition, 10, 104-114.

Nosofsky, R. M. (1988). Exemplar-based accounts of relations between classification, recognition, and typicality. Journal of Experimental Psychology: Learning, Memory, \& Cognition, 14, 700-708.

Nosofsky, R. M., \& Palmeri, T. J. (1997). An exemplar-based random walk model of speeded classification. Psychological Review, 104, 266-300.

PALMeri, T. J. (1997). Exemplar similarity and the development of automaticity. Journal of Experimental Psychology: Learning, Memory, \& Cognition, 23, 324-354.

Peterson, S. A., \& Simon, T. J. (2000). Computational evidence for the subitizing phenomenon as an emergent property of the human cognitive architecture. Cognitive Science, 24, 93-122.

Saltzman, I. J., \& Garner, W. R. (1948). Reaction time as a measure of span of attention. Journal of Psychology, 25, 227-241.

Sathian, K., Simon, T. J., Peterson, S., Patel, G. A., Hoffman, J. M., \& GRAFTON, S. T. (1999). Neural evidence linking visual object enumeration and attention. Journal of Cognitive Neuroscience, 11, 36-51.

ShIN, H. J., \& Nosofsky, R. M. (1992). Similarity-scaling studies of dot-pattern classif ication and recognition. Journal of Experimental Psychology: General, 121, 278-304.

Simon, T. J., \& VAishnaVi, S. (1996). Subitizing and counting depend on different attentional mechanisms: Evidence from visual enumeration in afterimages. Perception \& Psychophysics, 58, 915-926.

SLIWINSKI, M. (1997). Aging and counting speed: Evidence for processspecific slowing. Psychology \& Aging, 12, 38-49.

Spelke, E. S. (2000). Core knowledge. American Psychologist, 55, 1233-1243.

StARKey, P., \& CoOper, R. G. (1980). Perception of numbers by human infants. Science, 210, 1033-1035.

Trick, L. M., EnNs, J. T., \& Brodeur, D. A. (1996). Life span changes in visual enumeration: The number discrimination task. Developmental Psychology, 32, 925-932.

Trick, L. M., \& Pylyshyn, Z (1994). Why are small and large numbers enumerated differently? A limited-capacity preattentive stage in vision. Psychological Review, 101, 80-102.

Tuholski, S. W., Engle, R. W., \& Baylis, G. C. (2001). Individual differences in working memory capacity and enumeration. Memory \& Cognition, 29, 484-492.

van Oeffelen, M. P., \& Vos, P. G. (1982). A probabilistic model for the discrimination of visual number. Perception \& Psychophysics, 32, 163-170.

Warren, H. C. (1897). Studies from the Princeton Psychological Laboratory, VI-VII (VI. The reaction time of counting). Psychological Review, 6, 569-591.

Wender, K. F., \& Rothkegel, R. (2000). Subitizing and its subprocesses. Psychological Research, 64, 81-92.

Wolters, G., van Kempen, H., \& WiJlhuizen, G.-J. (1987). Quantification of small numbers of dots: Subitizing or pattern recognition? American Journal of Psychology, 100, 225-237.

Wundt, W. (1896). Grundriss der Psychologie. Leipzig: Engelmann.

(Manuscript received December 17, 2001; revision accepted for publication July 30, 2002.) 